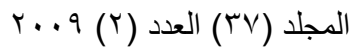
(ISSN 1815-316X)
مجلة زر اعة الر افدين

تأثير مواعيد الزراعة والرش بحامض الجبرليك في النمو الخضري وكمية ونوعية الحاصل لصنفين من

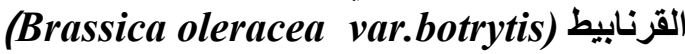

\author{
محمد طلال عبد السلام الحبار

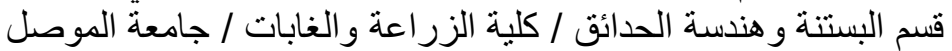

\section{الخلاصة}

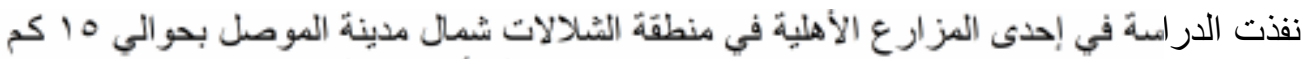

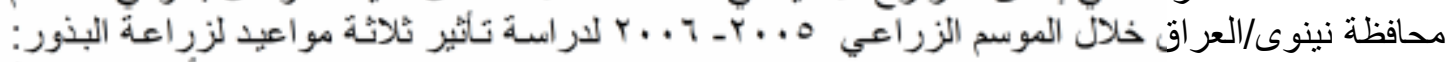

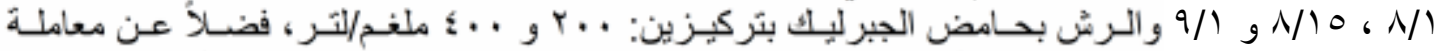

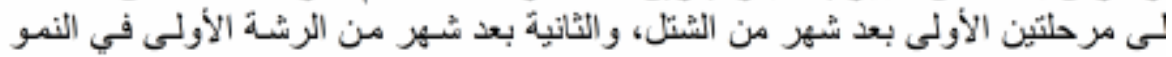

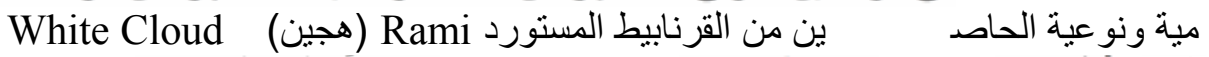

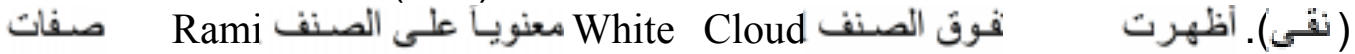

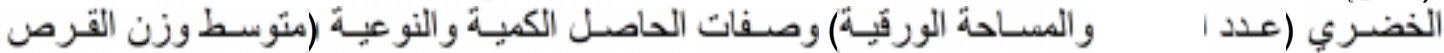

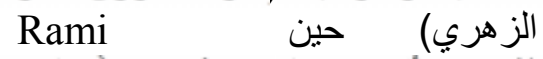

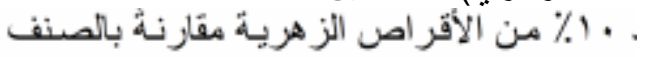

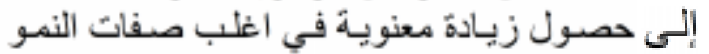

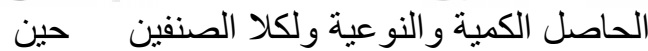

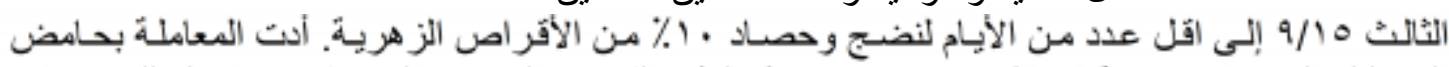

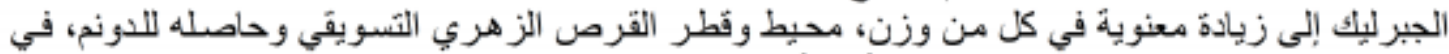

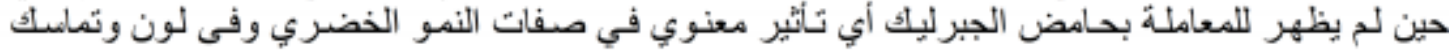
الز هري.

\section{المقدمة}

الصليبية Brassicaceae العديد من محاصبيل الخضر المهيمة | بأتى في مقدمتها

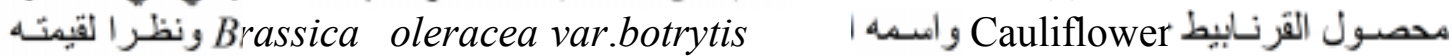

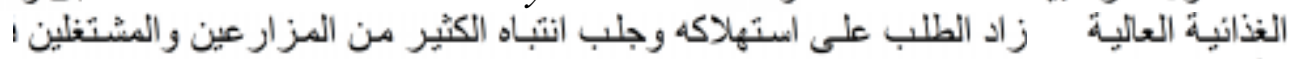

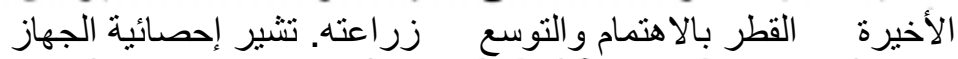

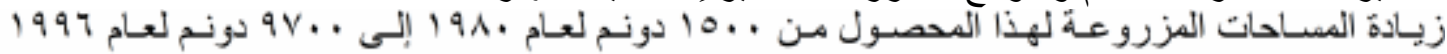

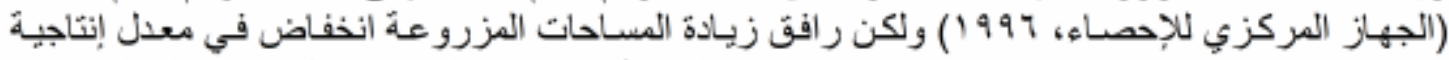

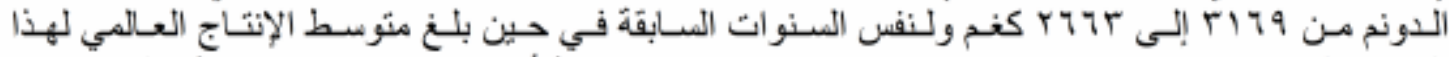

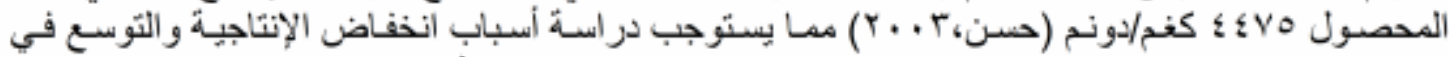

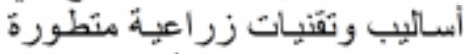

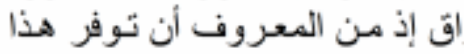

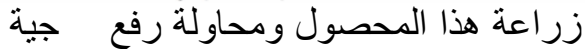

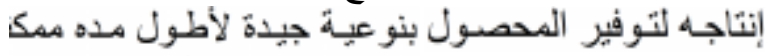

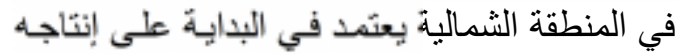

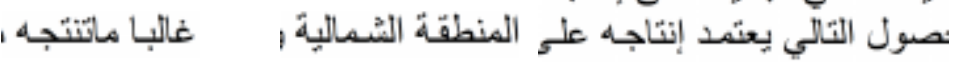

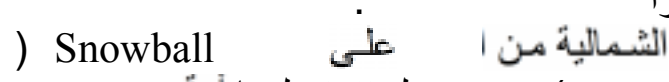

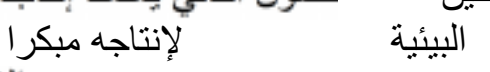

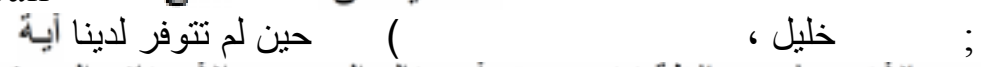

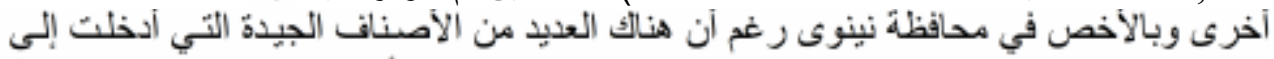

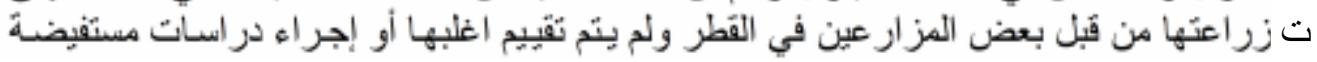

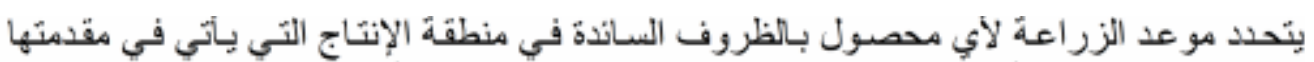
ليها.

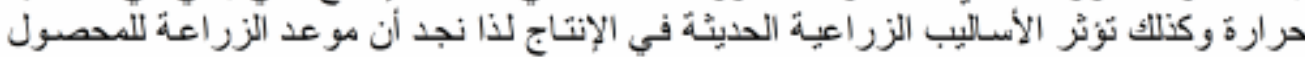

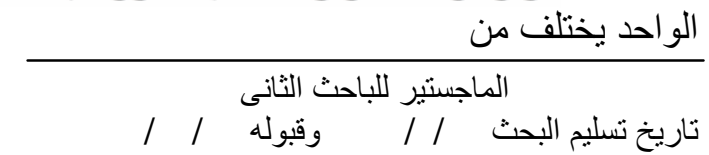




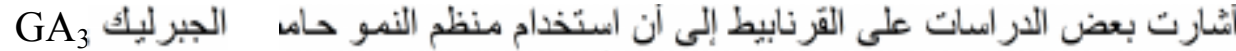

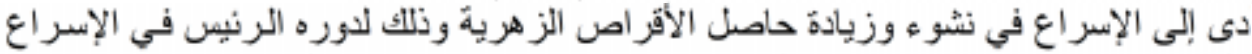

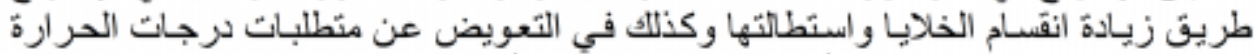
Abdalla) Vernalization

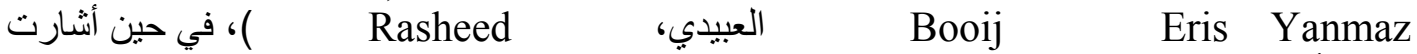

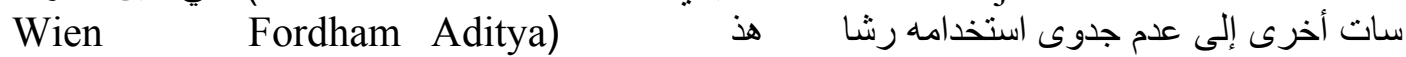

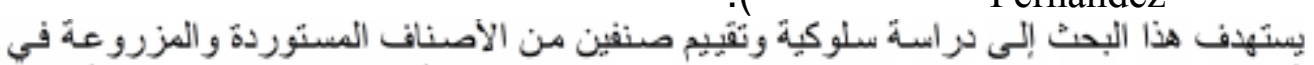

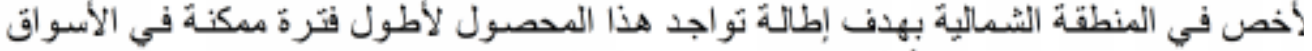

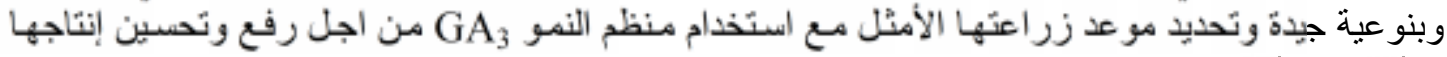

\section{مواد البحث وطرائقه}

نفذت الدر اسة في إحدى المزارع الأهلية في منطقة الثنالات شمال مدينة الموصل

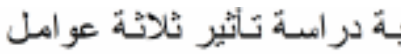

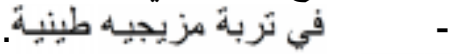

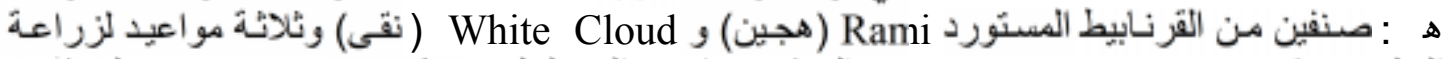

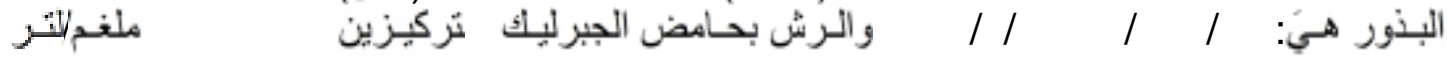
(Control)

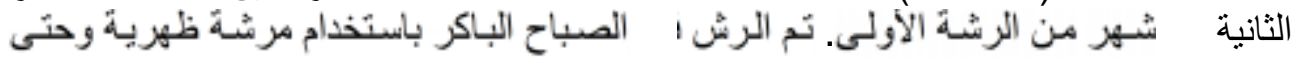
$\times \times$

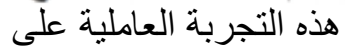
(Run off point)

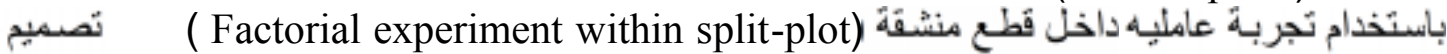

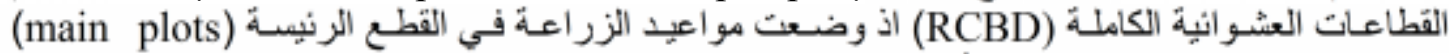

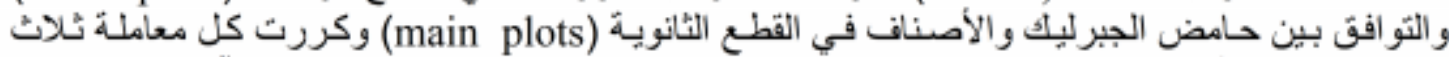

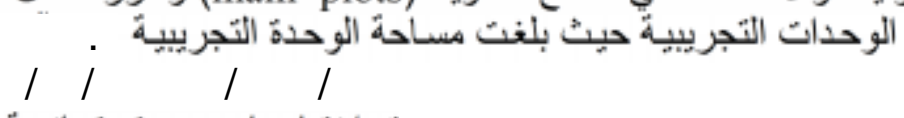

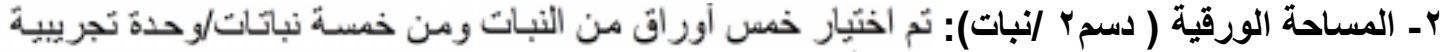

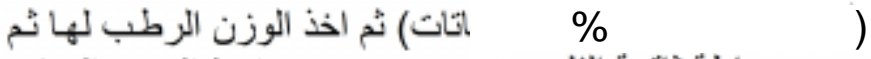

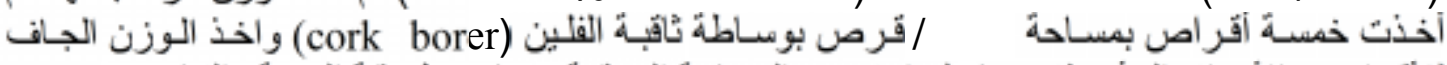

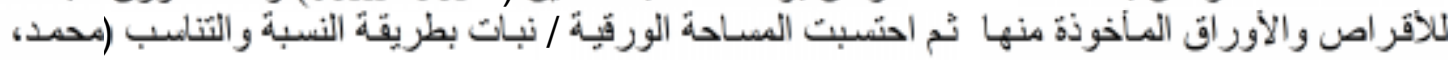

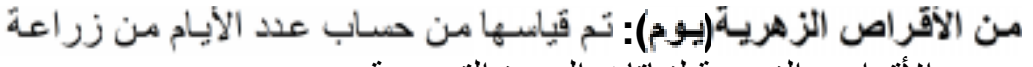

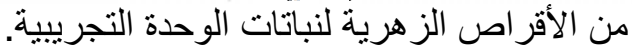

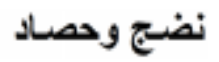

البذور ولحين نضج وحصاد 
ـ متوسط وزن القرص الزهري للحاصل التسويقي كفر: تم حسابها من الحاصل التسويقي للوحدة التجريبية

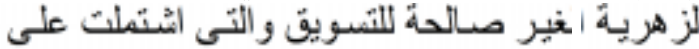

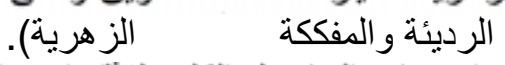

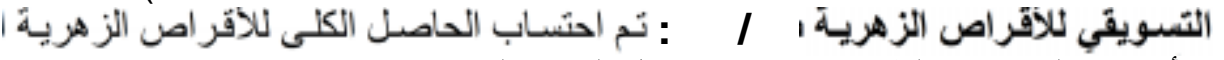
مقسومة على عدد الأقر اص لهاص الزها

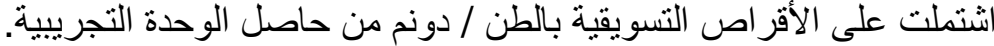

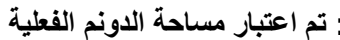

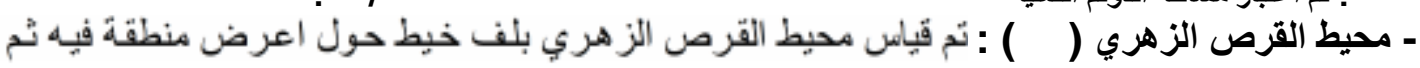
قياس طول الخيط بو الزوري

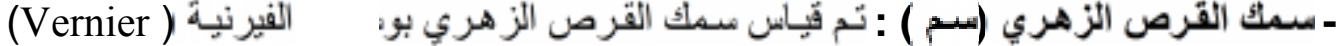

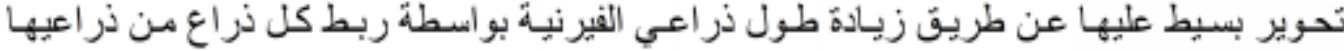

القرص الزهري ( ) ): تم إعطاء الدرجات الثَالبة للالالة على لون وتماسك

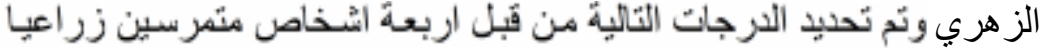
الزهري

القرص الزهري

$$
\text { متوسط التر اصيف التر اص }
$$

\author{
|بيض مصفر البيا \\ متوسط البياض ميفر \\ ابيض نامن \\ ابيض ناصع
}

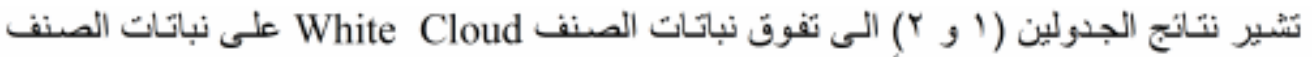

Rami

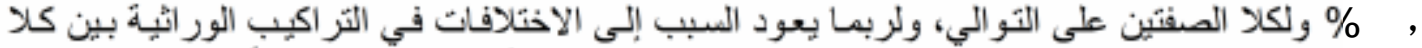

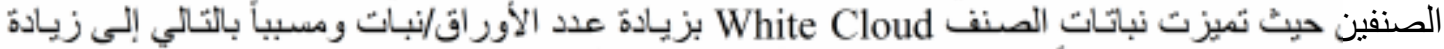

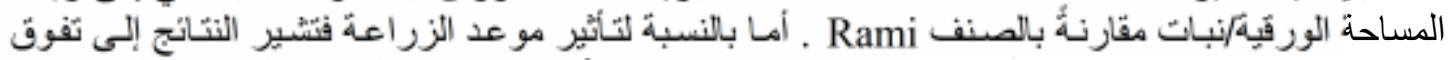

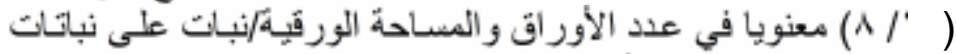

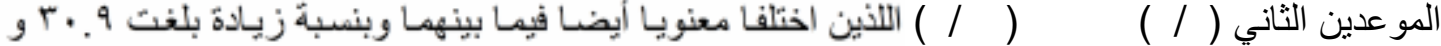

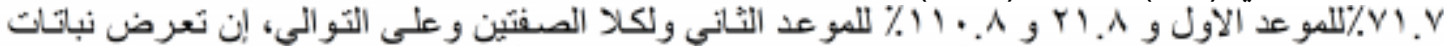

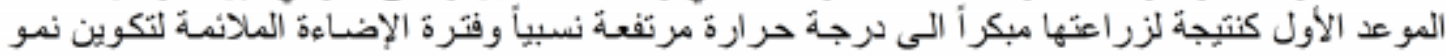

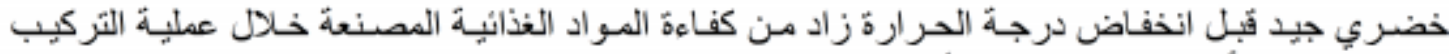

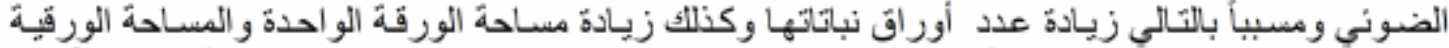

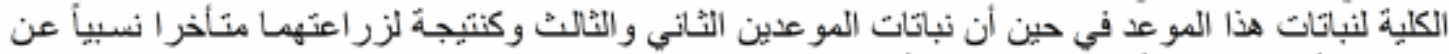

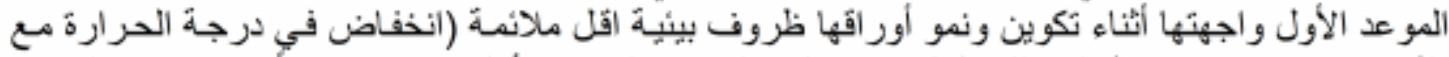

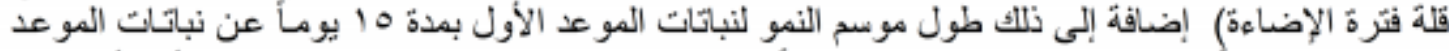

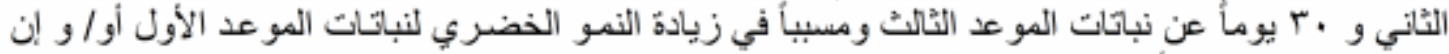

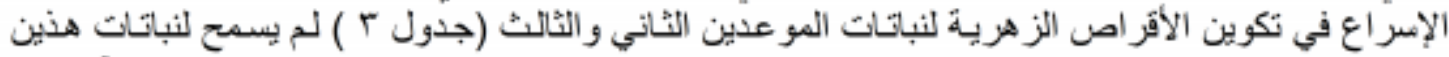

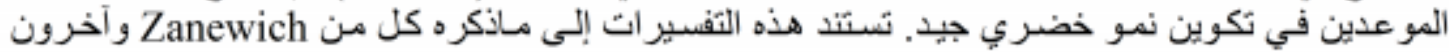

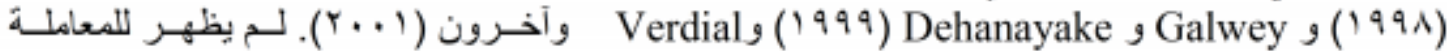

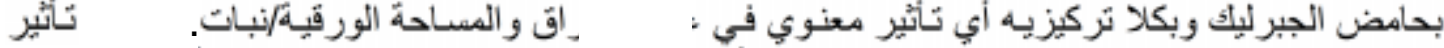

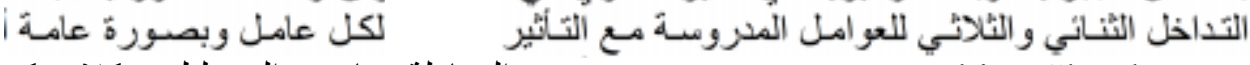

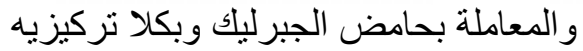

White Cloud

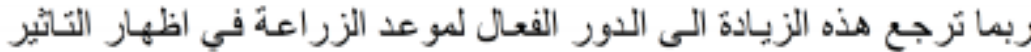

$$
\text { Rami }
$$

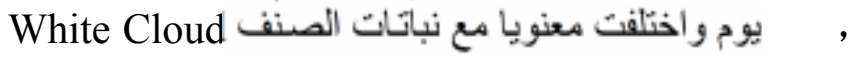

ومساحة ورقية/ الجبرليك

$$
\text { من أقر اصها الزهرية }
$$




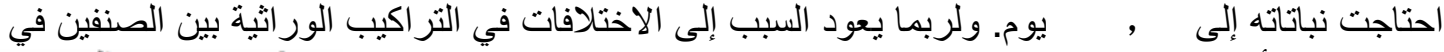
سر عة نشوء أقر اصهم الزهرية. حيث يعد الصنف Rami م

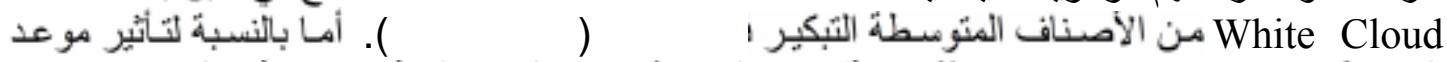

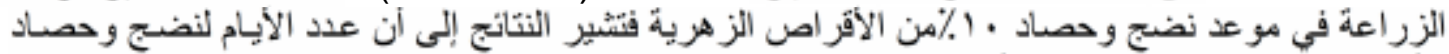

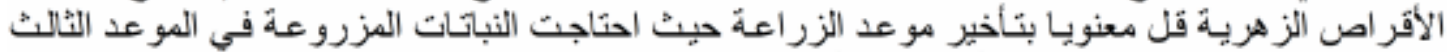

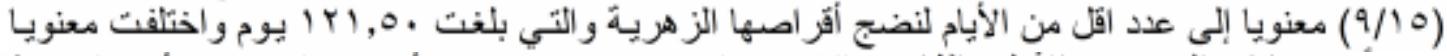

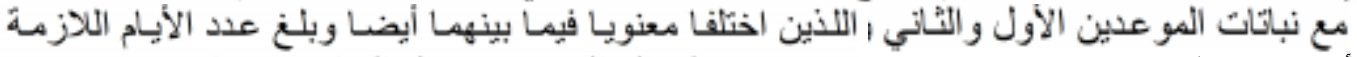

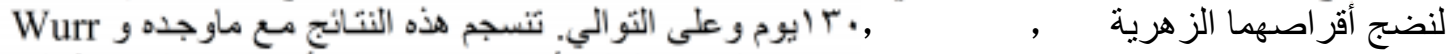
Fellows

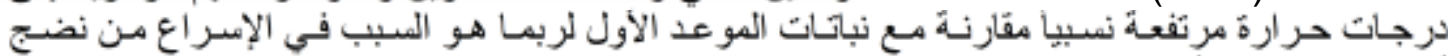

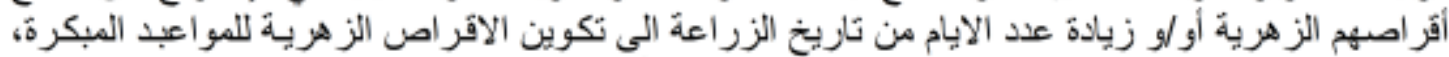

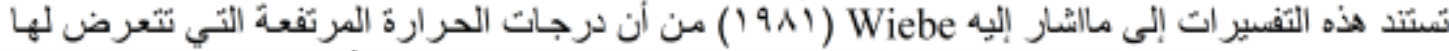

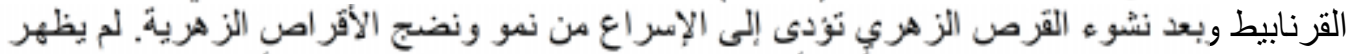

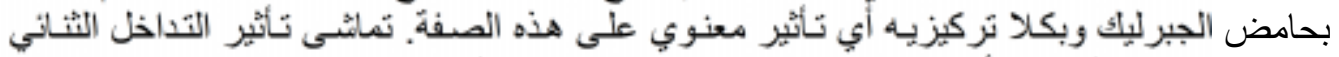

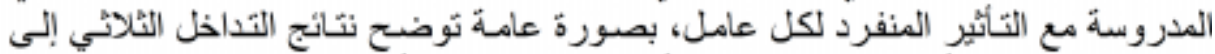

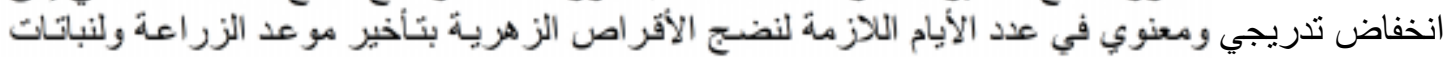

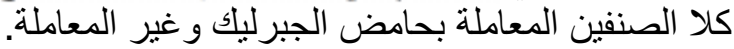
الز هري وحاصلة التشويقي معite Cloud لتأثنير

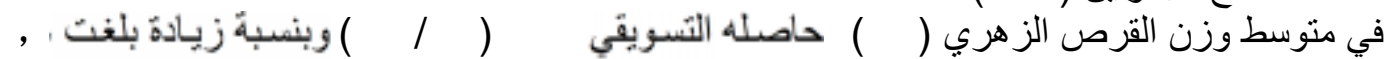

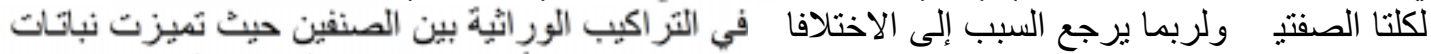
الجزئ White Cloud

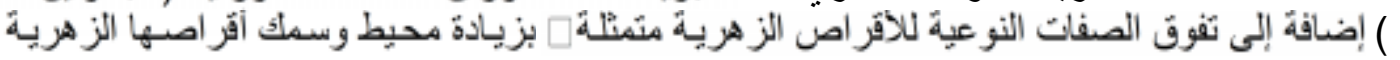

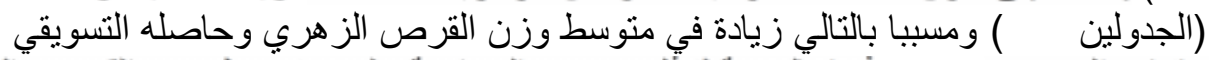

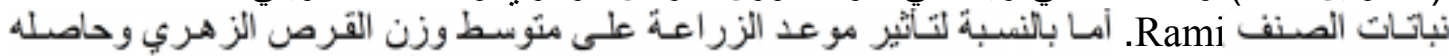

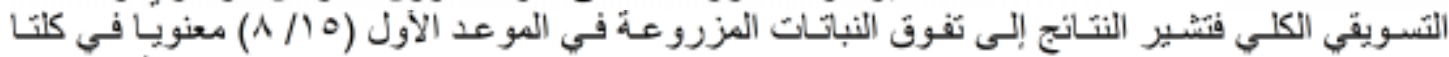

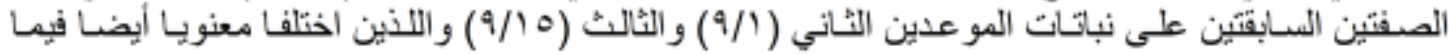

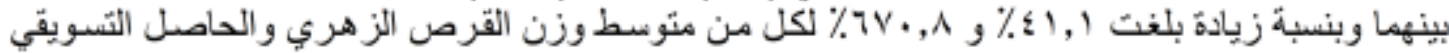

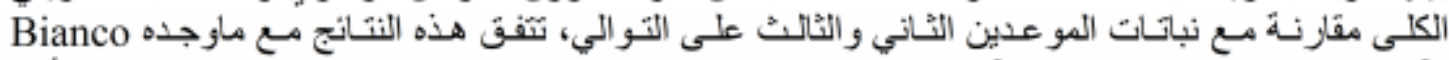

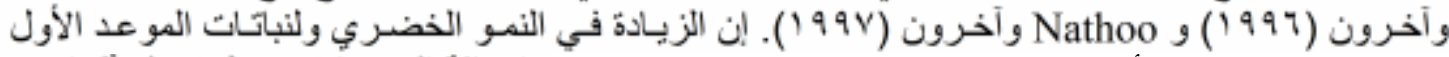

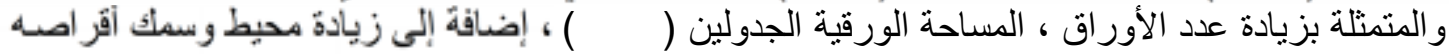

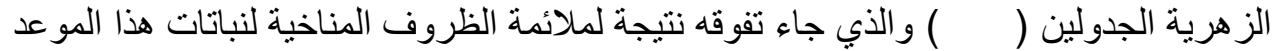

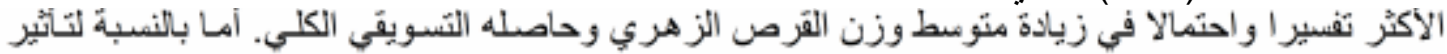

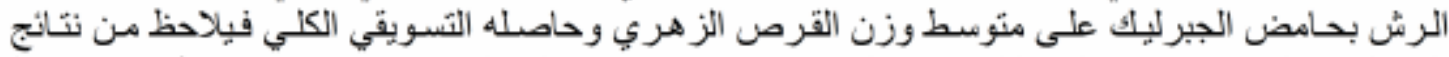

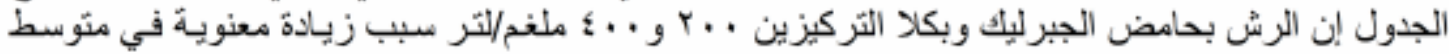

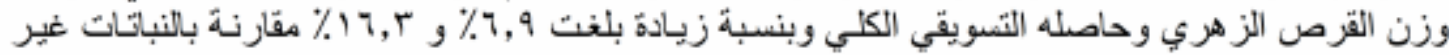

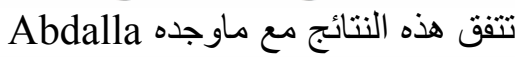

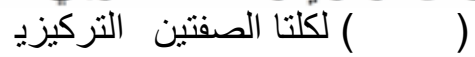

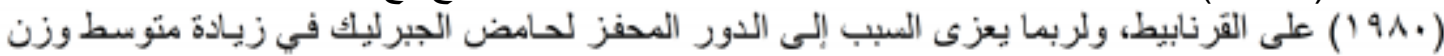

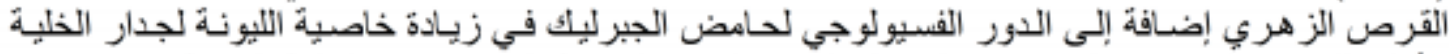

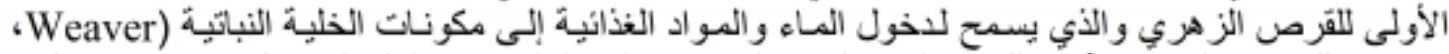

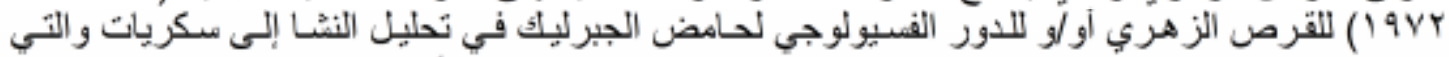

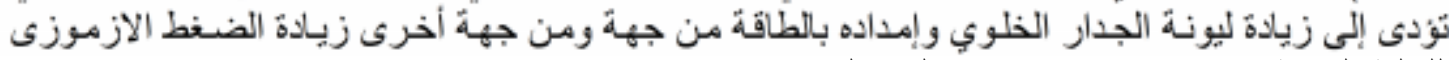

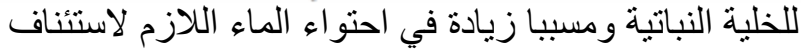

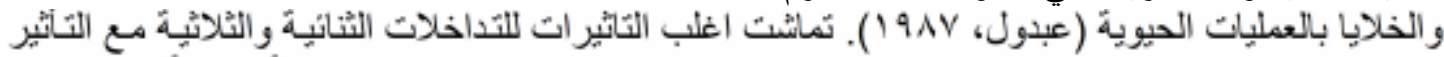

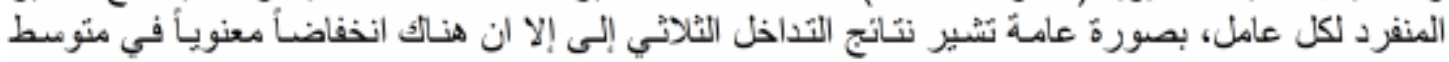




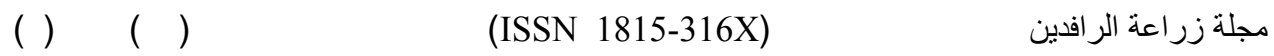

الزهري و الحاصل التسـويقي الكلى ولنباتُات كلا الصنفين المعاملـ وغبر |

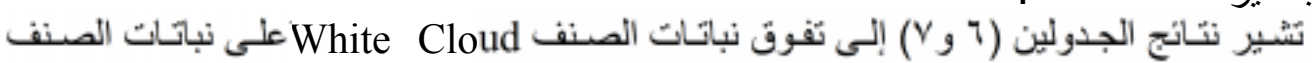

Rami

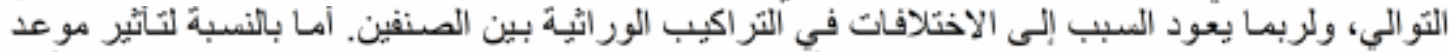

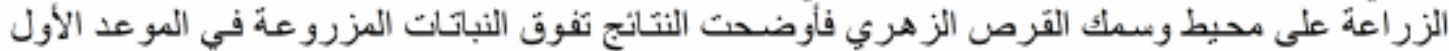

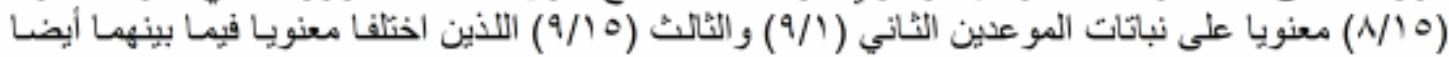

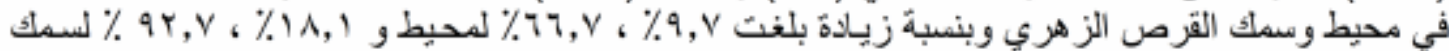

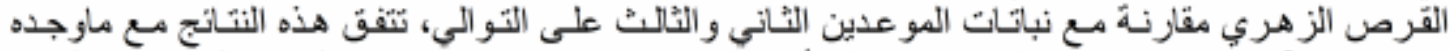

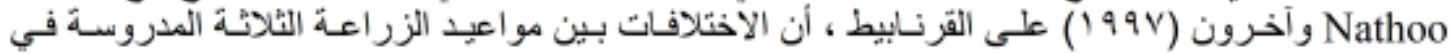

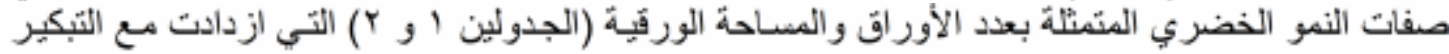

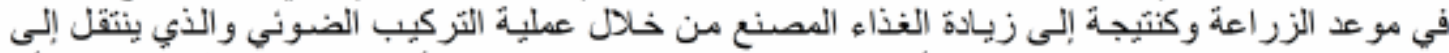

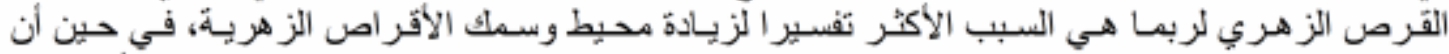

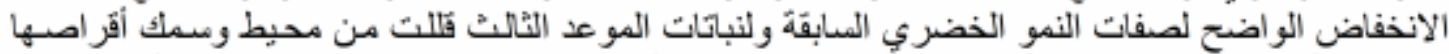

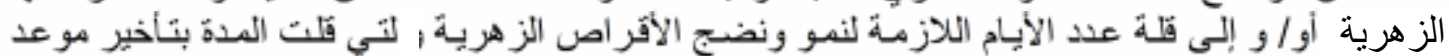

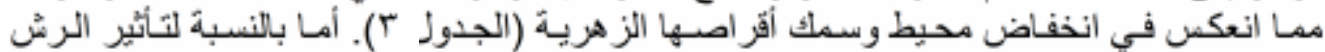

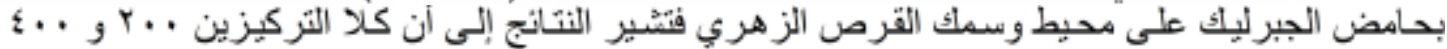

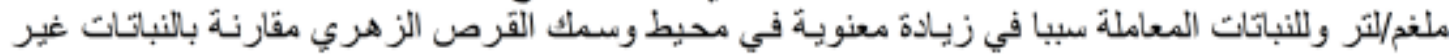

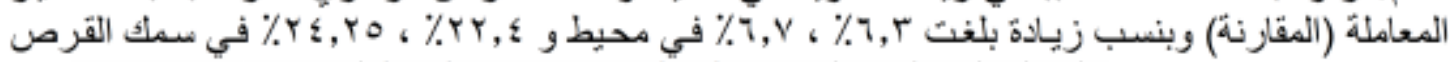

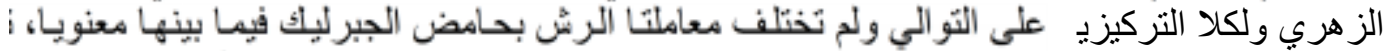

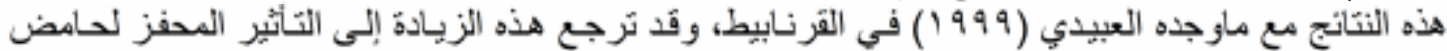

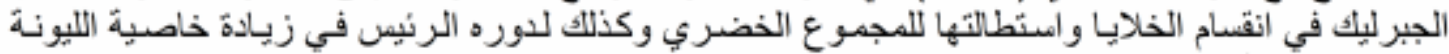

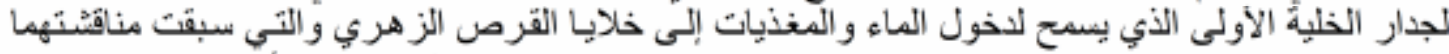

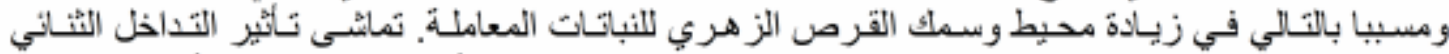

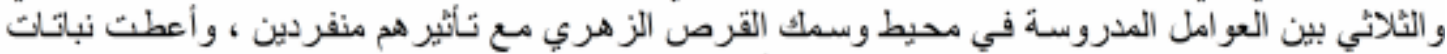

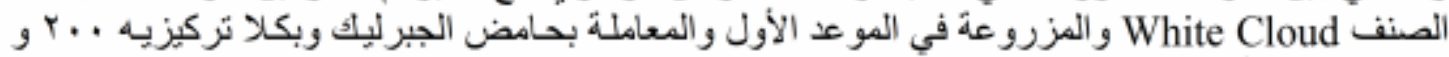

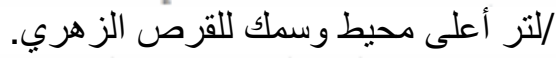

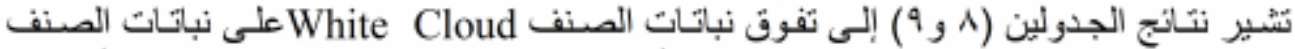

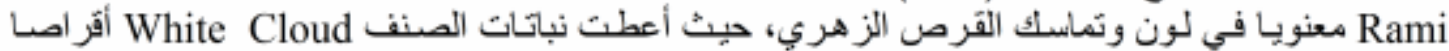

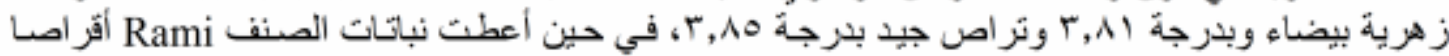

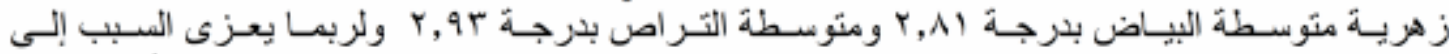

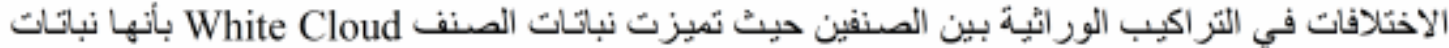

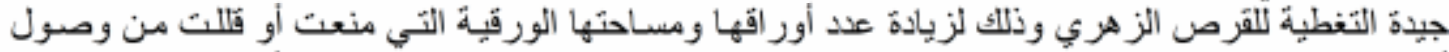

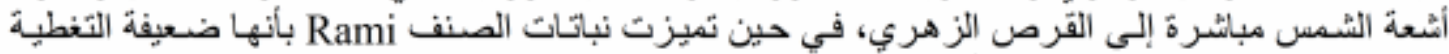

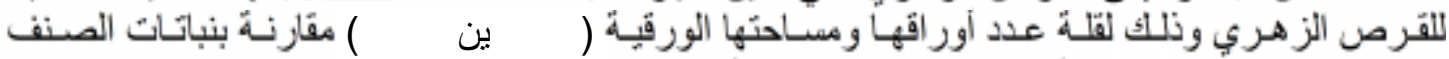

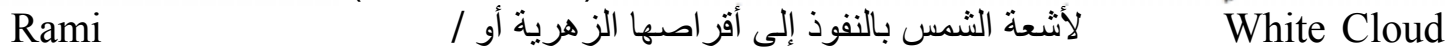

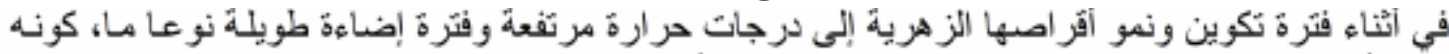

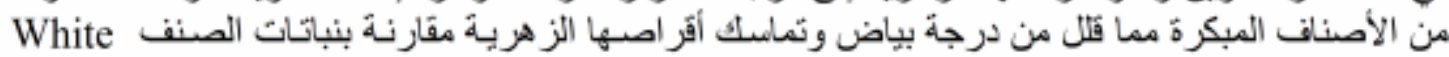

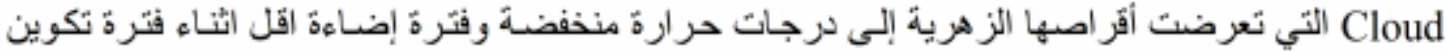

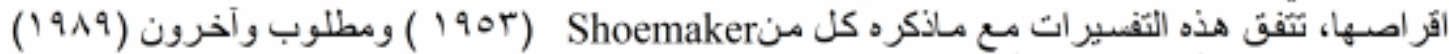

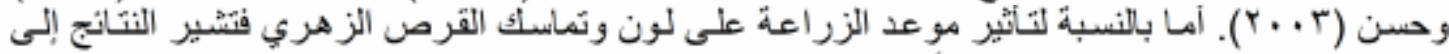

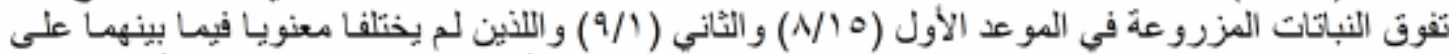

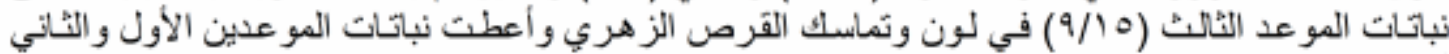

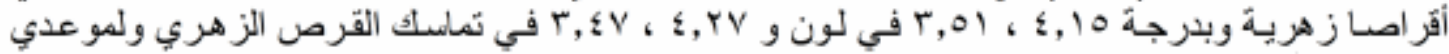

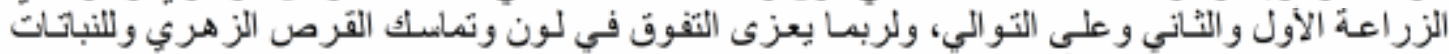

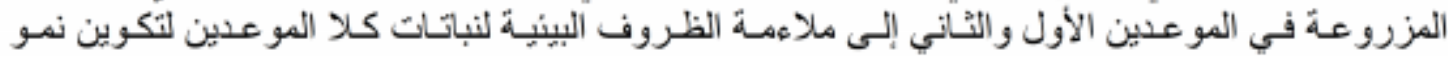




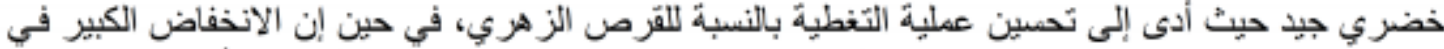

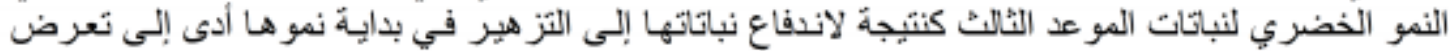

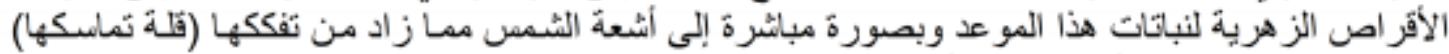

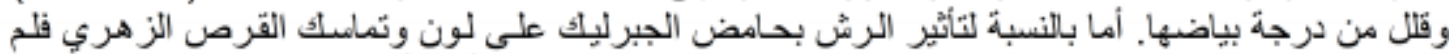

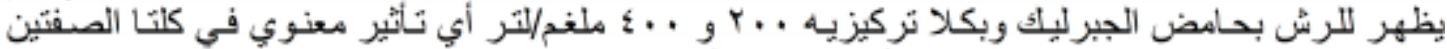

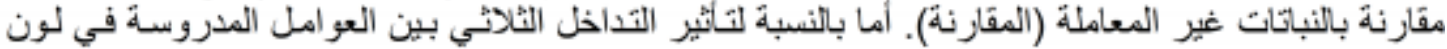

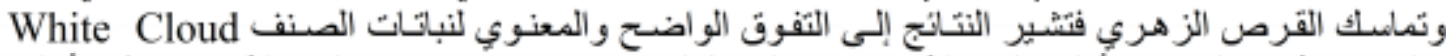

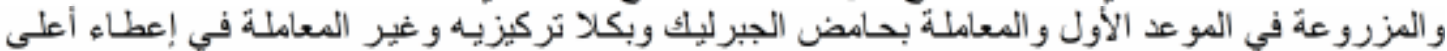

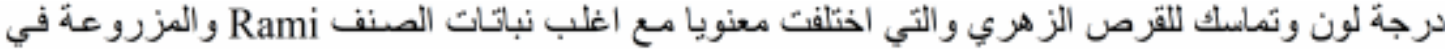

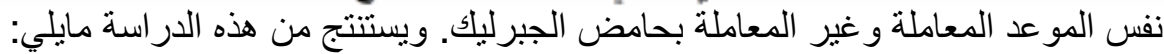

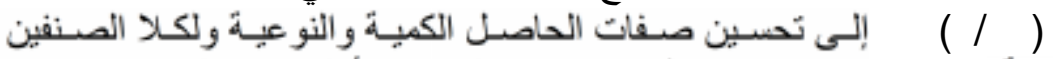
مواعبد زراعة مبكرةٌ عن الموعد الأول N/10 لغرض الإنثاج المستخدمين علبه بمكن التوصبية الئة المنطقة الثمالية.

ـ أظهر الصنف White Cloud التُفوف المعنوي الواضـح في جميع صفات الحاصل ونو عيته مقارنـة

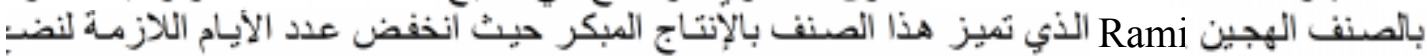

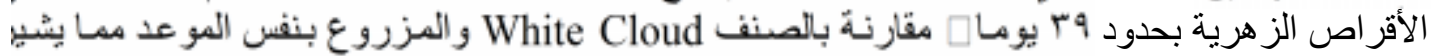

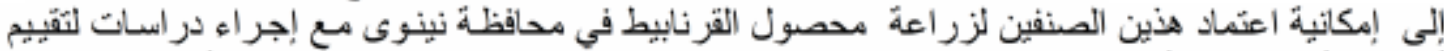

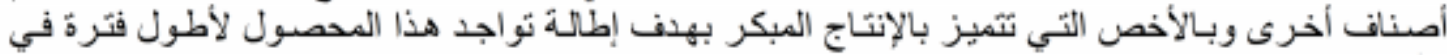

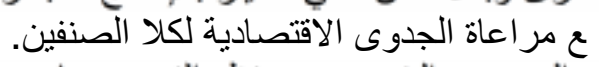

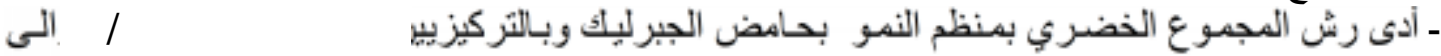

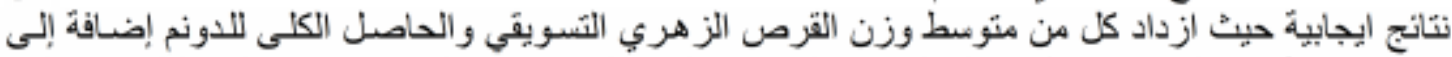

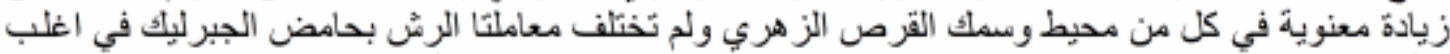

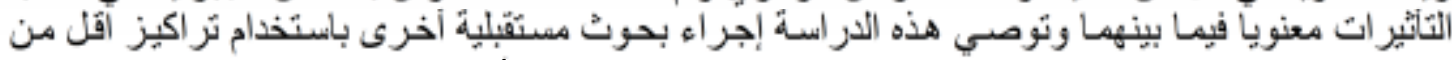
/لترمن حامض الجبرليك مع التوصية باستخدام منظمات نمو أخرى.

\title{
EFFECT OF PLANTING DATES AND FOLIAR APPLICATION OF GIBBERELLIC ACID ON VEGETATIVE GROWTH, QUANTITY AND QUALITY OF YIELD IN TWO CULTIVARS OF CAULIFLOWER (Brassica oleracea var. botrytis)
}

Mohammed Talal A. Al -Habar

Fathel Fatihe Rajab Ibraheem

Horticulture Dept., College of Agriculture and Forestry, Mosul univ., Iraq.

\begin{abstract}
This study was conducted in a private farm at Al-shallalat region approximately $15 \mathrm{~km}$ far from Mosul /Iraq during growing season of 2005_2006 to investigate the effect of three sowing dates $\left(15^{\text {th }} \mathrm{Aug}\right.$, 1st Sept. and $15^{\text {th }}$ Oct.), and the foliar application of $\mathrm{GA}_{3}$ at $(0,200$ and $400 \mathrm{mg} / \mathrm{l})$ on the vegetative growth, quantity and quality of curd yield in two Cauliflower cultivars Rami (Hybrid ) and White Cloud ( pure ). Treatment of $\mathrm{GA}_{3}$ were sprayed in two times, the first treatment was after a month of transplanting whereas the second treatment was done one month following the first spraying. The experiment involved 18 treatments arranged in a factorial experiment within split-plot using Randomized Complete Block Design with three replicates. The results could be summarized as follows:

1- White Cloud cv. Showed a significant increase in some vegetative growth (leaf number and leaf area), all yield quantity and quality characters (marketable curd weight, total marketable yield, curd circumference, curd thickness, curd color and
\end{abstract}




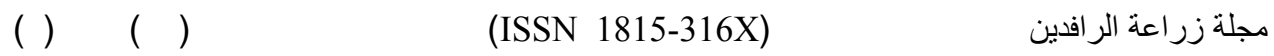

curd compactment) as compared with Rami cv. Whereas Rami cv. revealed a significant decrease in the number of days from sowing to maturation $10 \%$ of curd as compared with the White Cloud cv.

2- Early sowing date $\left(15^{\text {th }} \mathrm{Aug}\right)$ leads to a significant increase in all vegetative growth (leaf number and leaf area), all yield quantity and quality characters for both cultivars, whereas late sowing date $\left(15^{\text {th }} \mathrm{Oct}\right)$ leads to a significant decrease in the number of days from sowing to maturation $10 \%$ of curd in the two cultivars

3- Treatment with $\mathrm{GA}_{3}$ at two concentrations (200 and $400 \mathrm{mg} / \mathrm{l}$ ) showed a significant effects on average of marketable curd weight, total marketable yield, curd circumference and curd thickness, while it has no significant effects in the number of days from sowing to maturation $10 \%$ of curd, curd color and curd compactment. There where no significant effects between concentrations of $\mathrm{GA}_{3}$ in all the previous characters.

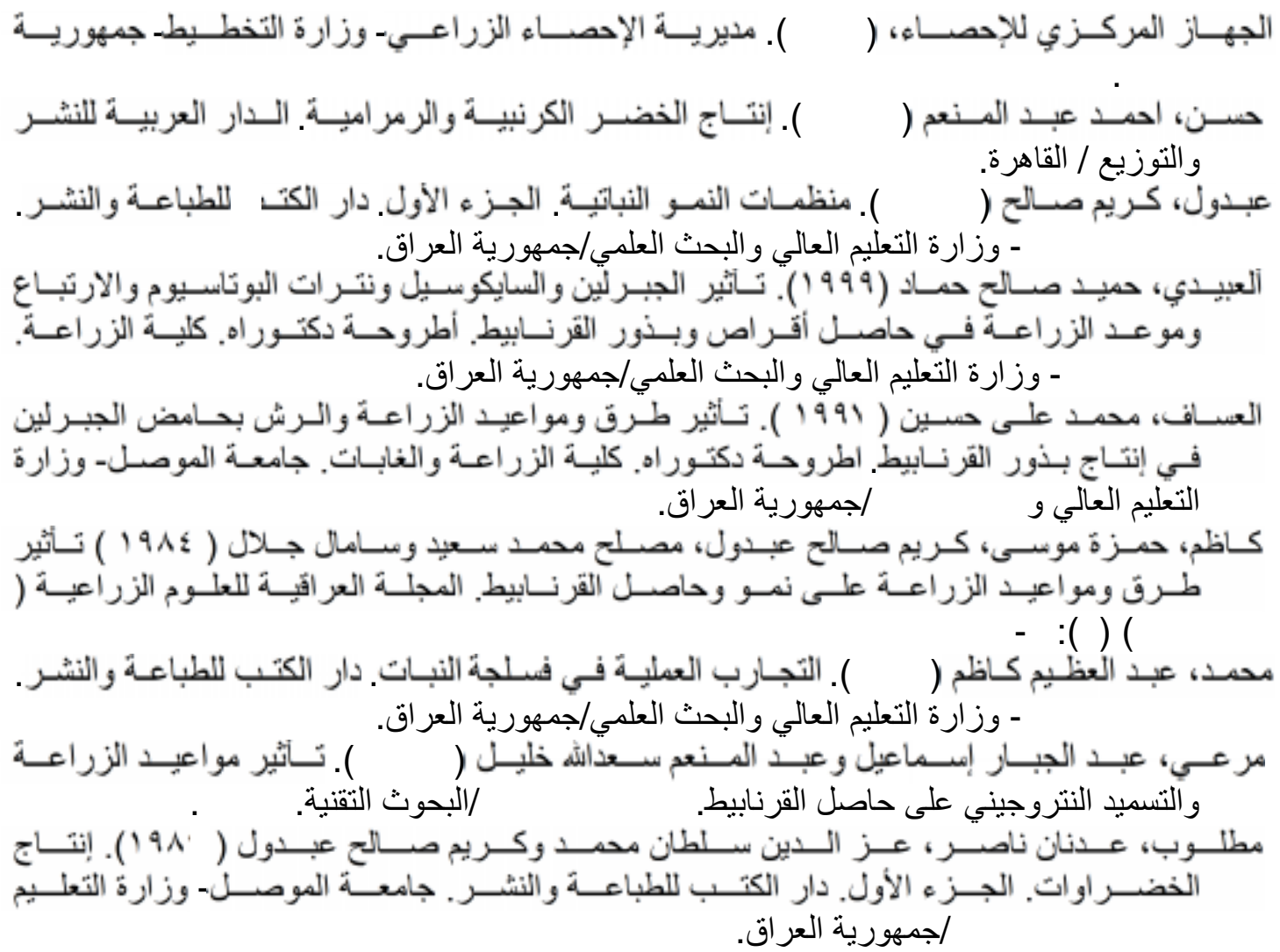

Abdalla, I. M.; R. M. Helal and M. E. S. Zaki (1980). Studies on the effect of some growth regulators on yield and quality of cauliflower. Annals of Agric. Sci. Moshtohor, 12: 199-208.

Aditya, D. K. and R. Fordham (1995). Effect of cold treatment and gibberellic acid on flowering of cauliflower. J. Hort. Sci. 70(40): 577-585.

Bianco, V. V.; G. Damato and R. Pomarica (1996). Sowing and transplanting dates in four Brassica cultivars-I. Sowing dates. Acta. Hort.(ISHI)407:299-304.

Booij, R. (1989). Effect of growth regulators on curd diameter of cauliflower. Scienitia Hort. (38): 23-32 . 


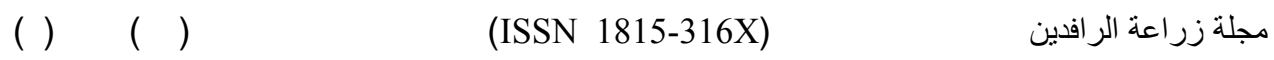

Fernandez, J. A.; S. Banon; J. A. Franco; A. Gonzalez and P. A. Martnez (1997). Effect of vernalization and gibberellins on curd induction and carbohydrate levels in the apex of cauliflower (Brassica oleracea var. botrytis). Scientia Hort. 70(2/3): 223-230.

Galwey, N. W. and S. R. Dehanayake (1999). Effect of interaction between lowtemperature treatments, gibberellin (GA3) and photoperiod on flowering and stem height of spring Rape (Brassica napas var. annua).Annals of Botany. 84: 321-327.

Verdial, M. F.; C. I. Antonio and L. S. Marcio (2001). Coincidence of flowering time and the productivity and quality of cauliflower hybrid seeds. Scientia. Agrigola. 58 (3): 533-534.

Nathoo, R.; M. N. Nowbuth and C. L. Cangy (1997). Production and varietial evaluation of cabbage and cauliflower. Agricultural Research and Extension Unit, Mauritius.

Rasheed, S. M. S. (2002). Effect of $\mathrm{GA}_{3}$ and Alar on curd and seeds yield of cauliflower (Brassica oleracea var. botrytis) grown in plastic house and in the field. M.Sc. Thesis. Hort. Dept, Agric. College. Dohuk Univ. Iraq.

Shoemaker, J. S. (1953). Vegetable Growing. ( $2^{\text {nd }}$ ed.). Jon Wily \& Sons., Inc., N. Y. 515 p.

Verdial, M. F.; C. I. Antonio and L. S. Marcio (2001). Coincidence of flowering time and the productivity and quality of cauliflower hybrid seeds. Scientia. Agrigola. 58 (3): 533-534.

Weaver, R. J. (1972). Plant Growth Substances in Agriculture W. H. Freeman and Company, Sanfrancisco . p. 494.

Wiebe, H. J. (1981). Influence of transplant characteristics and growing conditions on curd size. Acta Hort. 122: 99-105

Wien, H. C. and D. C. E. Wurr (1997). Cauliflower, Broccoli, Cabbage and brussel sprout.(C. F. Wien, H. C. (1997) The Physiology of Vegetable Crops. CAB International N. Y. USA. PP: 662).

Wurr, D. C. E. and J. R. Fellows (1984). Cauliflower buttoning the role of transplant size . J. Hort. Sci. 59(3): 419-429.

Yanmaz, R. and A. Eris (1984). Research on the possibility of early seed production in cauliflower (Brassica oleracea var. botrytis cv. Winner) and some properties of the seed. Ankara University Fenbilimeri Enstitusu (1984) . No. BB. 4 , p. 15pp.

Zanewich, K. P.; T. I. Poter and S. B. Rood (1998). Light intensity, gibberellins content and the resolution of shoot growth in Brassica. Planta. 207: 505-511. 